\title{
OGM Information des consommateurs et séparation des filières OGM/non-OGM
}

\section{GM Food Consumer information and separating GM from non-GM sector}

Oléagineux, Corps Gras, Lipides. Volume 7, Numéro 5, 399-403, Septembre - Octobre 2000, La filière, aujourd'hui, demain

Auteur(s) : Egizio VALCESCHINI, INRA, 16, rue Claude-Bernard, 75231 Paris Cedex 05.

Résumé : Un des problèmes au centre de la controverse sur l'utilisation des organismes génétiquement modifiés (OGM) dans l'agriculture et l'alimentation est celui de l'information des citoyens et des consommateurs. En première approche, deux positions radicalement opposées s'affrontent [1]. La première consiste à soutenir que l'étiquetage est inutile à partir du moment où la mise en marché du produit a été autorisée par les instances d'homologation sur la base d'une expertise scientifique. Garantissant l'innocuité, l'autorisation de mise en marché fournirait la seule information obligatoire véritablement pertinente pour les consommateurs : à savoir que le produit est sain1. Les tenants de cette position, notamment les États-Unis, voient donc dans I'obligation d'étiqueter un moyen de protectionnisme déguisé. La deuxième position consiste, au nom de l'intérêt des consommateurs, à demander que soit rendu obligatoire par la réglementation un étiquetage spécifique, qui permette de différencier nettement les produits issus d'OGM de ceux qui n'en sont pas. C'est la position récente de la Commission européenne depuis 1997 et le règlement novel foods sur les nouveaux aliments et les nouveaux ingrédients alimentaires. De cette manière, les consommateurs auraient la possibilité de faire leur choix et de manifester leur préférence en toute connaissance de cause. On considère implicitement que le marché fonctionnerait alors correctement et sélectionnerait, entre les produits transgéniques et les autres, ceux qui donnent le plus satisfaction aux consommateurs. Cette position a gagné du terrain sur le plan international, puisque plusieurs pays ont adopté récemment une réglementation sur l'étiquetage obligatoire des OGM (Japon, Australie, Suisse, etc.). La réflexion menée dans cet article s'inscrit dans le sens de cette dernière position, mais vise à poser plusieurs questions importantes qu'elle soulève2. L'argumentation en faveur d'un étiquetage distinctif repose sur le postulat que la présence ou non d'OGM est l'information pertinente pour le consommateur, celle qu'il recherche en priorité, avant les caractéristiques concernant la conservation, le goût du produit ou le respect de l'environnement par exemple. À supposer que cela soit établi, il reste à déterminer laquelle de l'allégation positive (du type " contient des OGM ») ou de l'allégation négative (du type " non-OGM ", " sans OGM " ou " sans utilisation d'OGM ») est la plus pertinente. Dans tous les cas, il est nécessaire de savoir s'il est possible, en termes de coûts et de fiabilité, de mettre en œuvre de manière efficace, un système de gestion de cette information sur la base d'une séparation des filières « avec » et « sans OGM ». II sera donc également nécessaire d'évaluer le coût de l'information fournie sur l'étiquette (le coût de la ségrégation des filières et le coût de la garantie que cette ségrégation est efficace), c'est-à-dire d'évaluer si les coûts de la traçabilité et des contrôles permettent d'assurer la crédibilité de l'allégation. Les enjeux de pertinence et de faisabilité se rejoignent à propos de la question de savoir 
si un simple étiquetage universel, clair et lisible peut suffire à susciter une offre et une demande qui satisfassent les consommateurs, ainsi que les attentes des citoyens, dans un contexte où, bien entendu, les pouvoirs publics appliquent des critères très stricts d'autorisation de mise sur le marché.

Summary : One issue raised by the use of GMO in agriculture and food is the one of information of citizens and consumers. The article first deals with the significance and limits of various labelling strategies. According to the position of the European authorities a labelling which differentiates GMO products. One other position would be to identify the "non-GMO" products. The article attempts to underscore the technical and economic regulations that could make credible a "non-GMO" labelling.

\section{ARTICLE}

\section{Quelle stratégie d'étiquetage en matière d'OGM ?}

\section{Quel objectif pour une stratégie d'étiquetage?}

Une des difficultés du débat sur l'étiquetage des produits transgéniques provient de la confusion entre le problème de la sécurité et celui de l'information. Le problème de la protection des consommateurs concerne la sécurité hygiénique et sanitaire du "mangeur ", l'exigence fondamentale étant la préservation de la santé. Cette question est aujourd'hui considérée, dans tous les pays industrialisés, comme relevant légitimement de l'activité réglementaire et répressive des pouvoirs publics. Cette légitimité repose sur le fait que la santé est considérée comme un bien public. L'État est reconnu comme l'instance supérieure seule à même de pouvoir engager sa responsabilité pour préserver et garantir efficacement la sécurité des citoyens, notamment en le préservant des falsifications. Un autre problème est celui de l'information de l'acheteur dans une économie marchande : I'objectif est d'assurer la loyauté des transactions (éviter aux consommateurs d'être victimes de tromperie) et de donner aux consommateurs la possibilité de choix en toute connaissance de cause. L'intervention réglementaire porte alors sur l'information, et se traduit notamment par des normes d'étiquetage sur les produits.

II faut souligner que, dès qu'un produit est autorisé à la vente, une information du type " produit avec OGM » apposée sur l'étiquetage n'apprend rien de plus au consommateur sur son innocuité. Certes une telle information lui permet de se déterminer de manière purement individuelle face à la présence d'OGM. C'est la logique même du marché qui s'applique : "j'achète ou je n'achète pas » et les produits non achetés sont de facto exclus du marché. Mais il faut insister : c'est l'analyse des risques qui doit montrer si des doutes subsistent ou non quant à la santé de consommateurs ou la qualité de l'environnement. Si la réponse est positive, l'utilisation des OGM doit être interdite. Ce n'est que si elle est négative que se pose la question du choix de la stratégie d'information des consommateurs.

La très grande majorité des consommateurs s'estiment sous-informés en ce qui concerne les enjeux des OGM. Cette opinion est partagée y compris par les consommateurs aux opinions favorables aux OGM. Une question importante apparaît : quel est l'objet du choix que l'on veut permettre aux consommateurs ? S'agit-il de choisir entre des produits différents, comme le choix entre des yaourts " classiques » et des yaourts allégés par exemple? 
Ou s'agit-il de permettre aux individus de se déterminer par rapport au choix d'une nouvelle technologie concernant les méthodes de production ? Si c'est un choix portant sur les méthodes de production, il est clair que l'étiquetage, obligatoire ou non, positif ou négatif, n'est certainement pas le seul moyen de communication permettant aux consommateurs de s'informer. Si on considère qu'il s'agit d'un choix citoyen, ce sont des procédures de débat et de concertation qu'il s'agit de mettre en œuvre [2].

Tout au long de ses évolutions récentes, objet d'enjeux et résultat de compromis entre les diverses parties en présence, la réglementation européenne en matière d'étiquetage des OGM entretient et souffre de ces multiples confusions entre sécurité et information, entre choix citoyen et choix consommateur, entre information sur les caractéristiques du produit et information sur les méthodes de production.

Initialement, dans le cadre de la réglementation novel food [3, 4], les nouveaux produits soumis à I'homologation, dont les produits issus d'OGM, sont identifiés à partir du concept de " l'équivalence substantielle ${ }^{3}$. Dans son essence, cette identification des produits est donc tout entière guidée par une perspective de protection de la sécurité des citoyens et non pas de différenciation des produits pour informer les consommateurs sur le marché. En 1997, quand les autorités européennes, pressées par les opinions publiques, décident de distinguer les produits OGM et d'informer les consommateurs sur la base d'une information spécifique sur l'étiquette, la solution adoptée est de donner une information sur les caractéristiques du produit final. En effet, l'étiquetage est rendu obligatoire seulement dans les cas où l'on peut distinguer dans le produit final une trace de la modification génétique utilisée au cours du procédé de production. Dans le même temps, la question est posée de savoir s'il ne faudrait pas également étiqueter les produits " sans OGM " ${ }^{4}$. Un dernier pas est réalisé par les derniers règlements en date (début 2000), qui correspondent à donner aux consommateurs une information sur les méthodes de production. Ils précisent, d'une part, qu'un aliment à destination humaine contient des OGM quand au moins un de ses ingrédients contient plus de 1 \% d'OGM (reg. 49/2000) et, d'autre part, que l'étiquetage des additifs et des arômes qui contiennent des OGM est rendu obligatoire (reg. 50/2000), par exemple la lécithine de soja contenant des OGM doit désormais être signalée.

\section{Quelle information pertinente sur l'étiquette?}

En matière de pertinence, il convient d'abord de savoir où s'établit la ligne de partage entre ce qui relève du citoyen et ce qui relève du consommateur. Clairement, les questions d'environnement et de santé publique sont des questions citoyennes qui relèvent de l'autorisation de mise sur le marché par les pouvoirs publics. La mise en place d'une double filière n'a donc de sens que si aucun danger manifeste, pour la santé ou pour l'environnement n'est avéré et qu'aucun risque possible n'est pressenti. Dans ce cas, le consommateur peut légitimement exercer son libre choix s'il désire pour lui et ses proches un surcroît de précaution, sans qu'il considère pour autant que les autres produits mettent en péril l'environnement ou la santé publique. La double filière est pertinente également si le refus des OGM renvoie à des problèmes d'éthique majoritairement reconnus comme relevant de la sphère privée. II en va de même sans doute pour ce qui est du refus de certains d'avoir des aliments " trop éloignés de la nature ». Bien évidemment, les problèmes d'ordre économique et en particulier le pouvoir qu'exercent quelques grandes entreprises transnationales sur l'amont des 
filières de produits contenant des OGM ne seront pas totalement résolus par la mise en place de la double filière. II ne fait pas de doute que l'opinion publique française sait faire le lien entre ses attentes en matière de qualité alimentaire, d'une part, et le poids et la souveraineté de l'ensemble de sa filière agro-alimentaire, d'autre part.

Rappelons d'abord que les consommateurs ont le sentiment d'être plutôt dans un monde de produits " sans OGM ». Un produit sans allégation ou à l'allégation non visible est considéré par la plupart comme un produit sans OGM.

Les comportements vis-à-vis des OGM, négatifs mais également hétérogènes, suggèrent qu'il existe aujourd'hui une place pour des produits sans OGM dans une logique de deux filières séparées. Cette séparation est en tout cas souhaitée par l'opinion ${ }^{5}$. Le désir d'information et de choix ne correspond pas toujours à un désir d'évitement systématique. Il peut renvoyer à une simple volonté de conserver un degré de liberté et de précaution sur le plan individuel.

Au niveau des opinions, la quasi-unanimité des consommateurs réclame un étiquetage systématique, crédible et très visible des produits contenant des OGM. Quatre-vingt-dix pourcent réclament une allégation positive. Cette demande dépasse donc largement les réactions d'hostilité. Si $75 \%$ souhaitent spontanément un étiquetage des produits " non-OGM », les consommateurs ouverts aux OGM sont moins favorables à un tel étiquetage. L'intérêt pour l'allégation négative se réduit au cours des entretiens et des réflexions : on assiste à un refus d'un monde a priori OGM face auquel il conviendrait de se protéger.

\section{Quelle qualification pour les produits en matière d'OGM ?}

Une qualification de type "issus d'une matière première non-OGM " désigne un produit intermédiaire ou final qui est obtenu à partir de matières premières ou de méthodes de production qui excluent toute utilisation d'OGM. II s'agit donc d'une obligation de moyens. Elle est la plus facilement utilisable et on comprend son avantage pour les producteurs et les distributeurs, car il s'agit d'une information relativement contrôlable à partir de la maîtrise des opérations clés tout au long des filières (en particulier l'approvisionnement en semences). L'IP (Identity Preservation) ${ }^{6}$, mise en avant par les producteurs américains, s'apparente à cette première qualification. On comprend l'insatisfaction qu'elle peut susciter auprès des consommateurs puisque, en définitive, elle ne donne pas de garantie quant à la présence ou non d'OGM sur le produit mis à sa disposition.

Une qualification de type " sans OGM » indiquerait qu'il n'y pas utilisation d'OGM, à quelque stade que ce soit de la filière de production, et que le produit final destiné aux consommateurs en serait totalement exempt ; on peut parler ici de " zéro OGM ». Une telle qualification allie étroitement obligation de moyens et obligation de résultats à chacun des stades de la filière considérée. Scientifiquement, une qualification "zéro OGM " est considérée comme illusoire, car il est impossible d'affirmer l'absence d'OGM. Par suite, un seuil correspondant à la limite de détection des techniques semble une proposition logique mais non pertinente. Un tel seuil permettrait d'indiquer la présence d'OGM mais il ne permettrait pas de dire en quelle proportion. Une telle stratégie demanderait des échantillons de taille très importante, ce qui la rendrait économiquement difficile. II est indispensable de fixer une limite de quantification (c'est-à-dire attribuer un pourcentage en OGM en référence aux ingrédients des produits). La limite de quantification actuellement proposée dans le cadre de la normalisation des méthodes de détection des OGM est de $0,1 \%$. Nous sommes là dans 
une qualification de type " non-OGM », qui signifie que les analyses n'ont pas détecté d'OGM dans le produit final délivré aux consommateurs.

Cependant, spontanément, les consommateurs ne perçoivent pas d'enjeu de seuil concernant les OGM (typiquement, ils ignorent, pour la plupart, les seuils relatifs aux produits bio). Une fois alertés sur ces enjeux, les consommateurs se portent spontanément vers un seuil « au niveau de détection ». Cette position n'est pas nécessairement reliée à des opinions et des comportements négatifs face aux OGM. Ainsi, certaines personnes en font plutôt un élément rassurant, une preuve de la bonne maîtrise technique de la filière OGM.

Un autre problème très important est celui du " périmètre du produit ", c'est-à-dire à l'inclusion ou non de produits dérivés tels que le papier blanchi à l'amidon de maïs, le papier d'emballage constitué en tout ou partie de produits issus d'OGM, l'inclusion ou non d'auxiliaires technologiques (enzymes issus de micro-organismes, acides aminés, etc.).

Pour définir un produit et une filière " non-OGM ", il est nécessaire de spécifier à la fois une obligation de moyens (à travers un cahier des charges) et une obligation de résultats (situation par rapport à un pourcentage admis d'OGM). II faut également considérer que, théoriquement, on peut identifier trois catégories de produits distincts : des produits qualifiés " d'OGM ", des produits qualifiés de " non-OGM » et des produits non qualifiés. Deux grandes possibilités apparaissent pour différencier les filières, compte tenu qu'il existe déjà en Europe une réglementation sur l'étiquetage positif des OGM.

La première possibilité suppose de définir un seuil (qui pourrait être identique à celui de l'étiquetage positif, à savoir $1 \%$ ) différenciant les produits qualifiés d'OGM des deux autres catégories de produits qui se différencieraient entre eux par leurs cahiers des charges. La deuxième possibilité suppose la définition de deux seuils qui permettraient de séparer les trois catégories de produits : le seuil réglementaire à la base de l'allégation " contient des OGM » (1\%) et un autre seuil correspondant à la qualification « non-OGM ». Pour être le plus bas possible, ce second seuil devrait correspondre à la limite de quantification (capacité de la méthode non seulement à détecter mais aussi à quantifier les OGM). Entre les deux seuils se placeraient les produits ne correspondant à aucune qualification. On conçoit que la première possibilité est la plus facile à réaliser puisque l'obligation de résultat correspond simplement au respect de la réglementation ; on conçoit aussi qu'elle peut être perçue comme mensongère. Fondée sur un seuil plus faible, la seconde possibilité est susceptible d'engendrer des coûts supplémentaires. Nous sommes en face d'un arbitrage du type plus ou moins de pertinence contre plus ou moins de faisabilité.

\section{Comment assurer la crédibilité de l'étiquetage en matière d'OGM ?}

\section{Quelle signalisation et quelle garantie?}

D'une manière générale, dans l'agro-alimentaire, la question de l'information se pose dans des termes particulièrement difficiles. Les acheteurs ne sont en mesure de connaître automatiquement ni le niveau de qualité des produits, ni la véracité des informations sur les caractéristiques des produits, d'autant plus que les caractéristiques des denrées alimentaires sont pour l'essentiel des « caractéristiques d'expérience ", observables seulement après l'achat, à l'usage (comme la tendreté de la viande par exemple), ou des " caractéristiques de confiance " non identifiables même après 
l'achat. C'est par exemple le cas lorsque la composante "sécurité " de la qualité ou, encore, la composition nutritionnelle d'un produit sont en cause. C'est également le cas lorsque l'on s'attache aux composantes éthiques, culturelles ou environnementales de la qualité : rien par exemple ne permet de discerner, au moment de la consommation, un bien dont les conditions de production sont particulières en ce qui concerne le bien-être des animaux, les droits des travailleurs, la protection de l'environnement, ou bien encore qui est produit sans recours à des procédés particuliers (biotechnologie, irradiation, etc.), d'un bien produit dans d'autres conditions. Ces caractéristiques relèvent clairement de l'aspect " confiance ". II est clair que c'est le cas des produits OGM. Les sens et la capacité personnelle d'expertise du consommateur sont insuffisants pour lui permettre d'évaluer si un produit contient ou non des OGM. Même si une information en la matière lui est fournie, il ne peut pas lui-même en vérifier l'exactitude ou simplement en évaluer la fiabilité. Le consommateur doit donc recourir à une information et bénéficier d'une garantie, résumées par les économistes dans le terme de "signal de qualité " ${ }^{7}$. Pour être crédible, c'est-à-dire digne de confiance, un signal de qualité demande que sa validité s'appuie sur un mécanisme de régulation endogène au marché, ou sur l'expertise d'une personne, d'un organisme ou d'une institution fiable et reconnue. Le problème qui se pose alors est celui de la nature du mécanisme de garantie à mettre en œuvre.

D'un point de vue théorique, deux mécanismes sont susceptibles d'assurer la crédibilité d'un signal de qualité : l'établissement d'une réputation et les systèmes de certification. La réputation, qui repose sur la construction d'une image de marque grâce à la répétition des achats, associe la qualité du produit au nom de celui qui le fabrique et/ou le vend. Le signal de qualité est alors inhérent au fonctionnement du marché : plus la réputation est grande, plus le préjudice sera élevé en cas de défaillance, car c'est la rentabilité des investissements consentis pour créer et entretenir l'image de marque qui est menacée. Les noms commerciaux et la marque commerciale constituent des cas types d'un signal de qualité endogène. Les systèmes de certification, quant à eux, crédibilisent un signal de qualité grâce à l'intervention d'une institution formelle, extérieure aux transactions marchandes, qui garantit la véracité de l'allégation. Ces institutions formelles (tierce partie ou État) engagent alors leur propre responsabilité, fondée sur leur capacité d'expertise, la fiabilité de leurs contrôles et, en particulier en ce qui concerne l'État, sur leur puissance de coercition. Ce système est adapté à des situations où il est nécessaire de cordonner les plans et l'activité de nombreux acteurs indépendants, ce qui est le plus souvent le cas dans les filières agricoles et alimentaires. Dans l'agroalimentaire, la tendance actuelle est la mise en œuvre de stratégies mixtes combinant réputation et certification ${ }^{8}$.

De manière opérationnelle, aux niveaux français et communautaire, trois grands types de stratégie de signalisation et de garantie des produits pour une filière qualifiée de "non-OGM " sont envisageables :

- la création d'une allégation mise à disposition des acteurs économiques, avec référence à une norme de produit et à une norme de traçabilité, et contrôle par une instance publique (DGCCRF $\left.{ }^{9}\right)$;

- la création d'un label spécifique, élaboré et homologué par une instance officielle (par exemple $\left.\mathrm{CNLCC}^{10}\right)$, avec une certification par un organisme tiers ; 
- la réservation de la mention « non-OGM » aux certifications de qualité officielles existantes ${ }^{11}$ ou à l'une d'entre elles.

\section{Quelles contraintes pour séparer les filières et tracer les produits ?}

Sur le plan technique, quel que soit le mécanisme de garantie choisi, la fiabilité de l'étiquetage en matière d'OGM implique la délimitation d'une filière séparée et un système de traçabilité des produits [5]. En effet, seule une séparation totale entre les filières de produits transgéniques et les filières sans transgènes pourrait permettre d'envisager un contrôle de l'absence/présence de transgène. Rappelons encore qu'il n'est pas concevable que ce contrôle soit absolu, au sens de garantir " zéro OGM » (cf. supra). Il convient de s'assurer de la possibilité de maintenir, tout au long de la filière " de la fourche à la fourchette ", la séparation des ingrédients. Pollution au champs, contamination fortuite en stockage ou en transport, mélange ou confusion de lots en production ou en distribution sont évidemment possibles et posent des questions d'organisation, de seuils, de traçabilité et de contrôle.

Les filières agro-alimentaires sont particulièrement complexes, du fait de la faible concentration et de la faible intégration verticale, de l'internationalisation des flux de matières premières (problème des importations de soja par exemple), de la multiplicité et du degré d'élaboration des produits aux différents stades de production et de distribution. Le système de traçabilité à concevoir pour garantir un produit " non-OGM » est tout particulièrement exigeant en techniques d'information et en organisation. En confrontant les différents types de traçabilité utilisés actuellement dans les industries manufacturières aux diagrammes des filières soja et maïs, il apparaît que, pour assurer la fiabilité d'un système de contrôle et la crédibilité d'une filière "garantie sans OGM ", il est nécessaire de concevoir un système de traçabilité générique qui combine trois types de dispositifs qui se renforcent les uns les autres :

- un dispositif de traçabilité de l'origine, qui définit précisément les caractéristiques d'origine dont il est nécessaire de suivre et de contrôler le déplacement ;

- des procédures de détection des OGM, afin de pouvoir systématiquement vérifier a posteriori la présence ou l'absence d'OGM ;

- un dispositif de traçabilité de processus (boucle de rappel), afin de réaliser un contrôle et de retrouver la source des produits suspects en cas de défaillance constatée (présence au-dessus du seuil).

Comme son nom l'indique, la traçabilité de l'origine implique que soient identifiés et suivis, dès leur origine et tout au long de la chaîne agro-alimentaire dans les transformations ou utilisations successives, les produits issus d'OGM qui entrent dans la composition du produit final. Dès le stade de la production agricole, d'organiser l'identification et la séparation des parcelles cultivées, des lots collectés et stockés. 
Il faut ensuite, aux stades aval, établir des procédures de séparation et d'identification des lots de produits, qui sont rendues compliquées par le fait que la plupart des activités de transformation alimentaire comportent aujourd'hui la désagrégation de produits initiaux (mouture du blé en farine par exemple) puis l'assemblage de différents produits (plats cuisinés tout prêts par exemple).

\section{Quelles sont les variables déterminantes des coûts de séparation et de traçabilité ?}

Sur le plan économique, la faisabilité d'une filière séparée commande de s'assurer que la différenciation des produits et la coexistence des filières n'occasionnent pas un surcoût dissuasif pour les consommateurs. II convient de "localiser " les sources d'éventuels surcoûts liés à la séparation des filières et à la traçabilité afin, d'une part, d'évaluer leur niveau et, d'autre part, d'émettre une hypothèse sur leur répartition au sein de la filière agro-alimentaire, en particulier la part qu'il reviendra aux consommateurs de supporter. La "localisation » de ces sources de surcoûts correspond à l'identification des points critiques où se situent les principaux risques de mélange nonOGM/OGM et des dispositifs à mettre en place pour les éliminer aux différents stades des filières. Sans prétendre ici être exhaustif ou très précis dans cette identification, il apparaît au moins quatre facteurs clés.

La production et la commercialisation des semences. À l'origine de la production agricole, par définition, les semences constituent un premier verrou excessivement important dans le phénomène de dissémination-contamination génétique. Traditionnellement déjà très contrôlée dans le cadre d'une certification, l'activité semencière devra encore accroître ses performances en matière de pureté variétale. Chaque augmentation de performance en la matière risque de se traduire par des surcoûts importants pour les producteurs de semences et les utilisateurs.

La localisation des cultures. Au niveau de la production agricole, les principales sources de risque de dissémination-contamination génétique sont : la localisation des parcelles, les pratiques agricoles et les récoltes au champ. Les distances d'isolement ne sont donc qu'un des éléments qui permettront de garantir que les contaminations ne dépasseront pas un certain seuil. La localisation géographique des cultures et leur espacement dans le temps sont des éléments à considérer dans la maîtrise des risques de dissémination. Cela peut conduire à dédier les cultures à certaines régions.

Des équipements spécifiques. Au niveau de la collecte et du stockage, dans le circuit qui va de la sortie du champ à la commercialisation des lots de grains en sortie des entreprises de collecte stockage (ECS), les risques de dissémination-contamination génétique sont liés au mélange des lots et à l'utilisation d'équipements communs pour le transport et les transferts, le séchage et le stockage. Une séparation efficace des récoltes " non-OGM » en utilisant des équipements dédiés (séchoirs, silos, etc.) risque de devoir être mise en place.

L'organisation de la récolte et du stockage. La taille optimale des lots « non-OGM » est un facteur important pour déterminer la faisabilité technique d'une telle filière. Elle détermine notamment les possibilités d'acquérir des équipements spécifiques et constitue l'un des facteurs de surcoût. Les entreprises de collecte et de stockage ont déjà une expérience de la gestion de ce type de risque dans le cas de filières de produits spécifiques (maïs semences, waxy, riche en huile, adapté au gavage, le soja semences ou alimentaire). II reste que la gestion du risque commercial dans le cadre d'une garantie d'absence d'OGM sera beaucoup plus contraignante. 
Sur le plan économique, la mise en œuvre d'une filière séparée et de la traçabilité indispensable à la crédibilité d'un étiquetage spécifique va certainement se traduire par l'apparition de surcoûts. On peut raisonnablement penser qu'ils seront variables selon les stades de la filière mais que, en tout état de cause, ils seront plutôt concentrés aux stades en amont. Si tel est effectivement le cas, compte tenu de la structure de la chaîne de valeur de la filière agro-alimentaire, cela signifierait que les surcoûts se dilueraient au fur et à mesure de la descente de la filière, pour atteindre un faible niveau au stade de la consommation.

II reste que, pour évaluer la faisabilité technique et économique d'une filière séparée garantissant des produits " non-OGM ", des variables importantes restent aujourd'hui indéterminées ou instables. Le montant des coûts va notamment dépendre :

- de la valeur du seuil d'allégation " non-OGM » (plus le seuil sera faible plus le coût de l'échantillonnage sera élevé et plus les coûts de déclassement des produits risquent d'être élevés) ;

- du nombre et des types de produits inclus dans le cahier des charges définissant un produit " nonOGM » qui devront être analysés (par exemple, prise en compte ou non des auxiliaires de fabrication, des additifs) ;

- du nombre de variétés OGM autorisées à la culture en Europe et des surfaces effectivement cultivées ;

- du contrôle documentaire de la traçabilité (plus ou moins précis et systématique, il nécessitera d'être plus ou moins complété par un dispositif d'analyse) ;

- des volumes de produits concernés (plus la taille du marché des produits garantis « non-OGM).

${ }^{1}$ Dans la même optique, une position moins extrême serait de considérer qu'un avertissement du type " peut contenir des OGM » serait acceptable. Mais cette mention pourrait avoir un effet pervers dans la mesure où elle pourrait donner à penser que le produit est potentiellement dangereux, alors que la procédure de mise en marché garantit que ce n'est pas le cas.

2 Les arguments développés dans cet article s'appuient pour l'essentiel sur les résultats intermédiaires de l'étude INRA "Pertinence et faisabilité d'une filière sans OGM » et, notamment, ceux de l'équipe de Bernard Ruffieux sur l'analyse économique du comportement et de l'opinion des consommateurs. L'auteur le remercie tout particulièrement ainsi que tous les participants à ce programme pour leurs apports. Il conserve l'entière responsabilité de la rédaction de ce texte.

${ }^{3}$ "Les procédures d'évaluation des risques et d'autorisation de mise en marché " reposent sur la comparaison des nouvelles denrées alimentaires avec les aliments (ou les ingrédients) existants et considèrent de la même manière la nouvelle denrée et l'aliment traditionnel lorsqu'aucune différence significative n'a été mise en évidence en matière de santé et de sécurité (aspects toxicologiques et nutritionnels). La notion " d'équivalent substantiel " est issue des travaux de la FAO, de I'OMS et de I'OCDE. Elle a été élaborée pour une raison bien expliquée par Gérard Pascal (OCL, 1997 : 241) : « l'évaluation de la sécurité n'est pas une évaluation toxicologique. (...) On ne dispose pas actuellement de méthodologie satisfaisante susceptible de tester sur les animaux de laboratoire des aliments et encore moins une alimentation. (...) Si un nouveau constituant alimentaire est démontré équivalent en substance à un aliment ou à un composant existant, cette 
relation d'équivalence est également valable sur le plan de la sécurité. Autrement dit, l'aliment ou le constituant nouveau sont considérés comme aussi sûrs (ou aussi peu sûrs) que l'aliment ou le constituant traditionnel. La sécurité de ce dernier est évaluée sur la base de son utilisation de longue date sans conséquences négatives sur la santé ».

${ }^{4}$ Selon Emma Bonino (1998 : 9), alors membre de la Commission européenne, chargée de la politique des consommateurs, il s'agit "d'aller plus loin pour respecter le droit fondamental des consommateurs de choisir les produits qu'ils achètent. La commission étudie les modalités possibles d'une "gamme de produits sans OGM", c'est-à-dire d'une gamme de produits qui ne contiennent pas d'organismes génétiquement modifiés ».

${ }^{5}$ Notons toutefois qu'il est difficile de globaliser à l'ensemble des comportements alimentaires des résultats qui sont par construction locaux, puisque effectués sur quelques produits.

${ }^{6}$ L'IP repose principalement sur une semence certifiée obtenue sans transgenèse, la spécialisation des régions de culture, la séparation des flux depuis la récolte jusqu'à la livraison des grains aux utilisateurs.

${ }^{7}$ Nous définissons un signal de qualité comme un résumé d'information qui apparaît sur un produit, ou une famille de produits, sous la forme d'un nom, d'un logo, d'un signe, etc., permettant aux consommateurs d'identifier et de distinguer le produit. Cette définition est plus restrictive que celle utilisée couramment par l'économie industrielle qui étudie d'abord le rôle du prix comme un signal de qualité.

${ }^{8}$ Un certificat officiel de qualité, comme le Label rouge par exemple, cherche à la fois à développer une réputation liée à une image de marque et une garantie officielle. Les situations de crise et d'incertitude forte sur la qualité suscitent de telles stratégies mixtes pour renforcer la crédibilité d'un signal de qualité ; c'est le cas par exemple en France, du sigle «VBF » (Viande bovine française) et du logo " CQC » (Certification qualité contrôlée) mis en place récemment dans le secteur de la viande bovine.

${ }^{9}$ Direction générale de la consommation, de la concurrence et de la répression des fraudes.

${ }^{10}$ Commission nationale des labels et des certifications de conformité.

${ }^{11}$ Appellation d'origine contrôlée, Label rouge, Certificat de conformité de produit, logo Agriculture biologique. 


\section{REFERENCES}

1. VALCESCHINI E, GOZLAN E, RAYNAUD E (1999). Quelle stratégie d'information pour les consommateurs ? Risques, $38: 73-8$.

2. BARBIER M, JOLY PB (2000). Sécurité alimentaire : quels enseignements pour les filières agroalimentaires. DEMETER 2001, 73-138.

3. COZIGOU G (1997). Un nouveau cadre juridique pour les applications biotechnologiques dans le secteur alimentaire : le règlement Nouveaux aliments. Revue du Marché unique européen, 2 : 67-80.

4. THIEFFRY P (1999). Le droit des OGM : précaution et information. Risques, 38 : 91-6.

5. DATTÉE Y, et al. (1998). Comment assurer la traçabilité des OGM et des produits issus d'OGM ? In : Organismes génétiquement modifiés à I'INRA. Environnement, agriculture et alimentation. Paris : INRA.

BONINO E (1998). L'Union européenne et la sécurité alimentaire. Revue du Marché unique européen, $4: 5-10$.

PASCAL G (1996). Comment garantir la sécurité du consommateur ? Rôle de la réglementation alimentaire. Cahiers Agricultures, 5 : 326-30.

PASCAL G (1997). La stratégie d'évaluation de la sécurité alimentaire des plantes transgéniques. OCL, $4: 241-4$.

VALCESCHINI E (1998). L'étiquetage obligatoire des aliments est-il la meilleure solution pour les consommateurs ? In : Organismes génétiquement modifiés à I'INRA. Environnement, agriculture et alimentation. Paris, INRA. 